KEK-TH-698

KIAS-P00028

OCHA-PP-160

hep-ph/0005313

October 29, 2018

\title{
Measuring the Higgs CP Property through Top Quark Pair Production at Photon Linear Colliders
}

\author{
Eri Asakawa ${ }^{1}$, S.Y. Choi ${ }^{2}$, Kaoru Hagiwara ${ }^{3}$ and Jae Sik Lee ${ }^{4}$ \\ ${ }^{1}$ Graduate School of Humanities and Sciences, Ochanomizu University, \\ 1-1 Otsuka 2-chome, Bunkyo, Tokyo 112-8610, Japan \\ ${ }^{2}$ Department of Physics, Chonbuk National University, Chonju 561-756, Korea \\ ${ }^{3}$ Theory Group, KEK, Tsukuba, Ibaraki 305-0801, Japan \\ ${ }^{4}$ Korea Institute for Advanced Study, Seoul 130-012, Korea
}

\begin{abstract}
We present a model-independent study of the effects of a neutral Higgs boson without definite $\mathrm{CP}$-parity in the process $\gamma \gamma \rightarrow t \bar{t}$ around the mass pole of the Higgs boson. Near the resonance pole, the interference between the Higgs-exchange and the continuum amplitudes can be sizable if the photon beams are polarized and helicities of the top and anti-top quarks are measured. Study of these interference effects enables one to determine the CP property of the Higgs boson completely. An example of the complete determination is demonstrated in the context of the minimal supersymmetric standard model.
\end{abstract}




\section{Introduction}

Search for Higgs bosons and precise measurements of their properties such as their masses, the decay widths and the decay branching ratios [1] are among the most important subjects in our study of the electroweak symmetry breaking. In the standard model (SM), only one physical neutral Higgs boson appears and its couplings to all the massive particles are uniquely determined. On the other hand, models with multiple Higgs doublets have neutral Higgs bosons of definite CP parity as well as charged Higgs bosons, if CP is a good symmetry. If CP is not a good symmetry of the symmetry breaking physics, these neutral Higgs bosons do not necessarily carry definite CP parity.

The CP-violating interactions beyond the Kobayashi-Maskawa mechanism and their consequences at high-energy colliders have been intensively studied. This is motivated in part by the search of an efficient mechanisms of generating the cosmological baryon asymmetry at the electroweak scale [2]. An extended Higgs-boson sector, as predicted by many extensions of the SM such as the minimal supersymmetric SM (MSSM), can provide such CP-violating interactions in a natural way. One attractive scenario is to make use of explicit CP violations in the MSSM Higgs sector [3, 4] which are induced through loop corrections with complex supersymmetric parameters in the mass matrices of the third generation squarks. Such interactions cause mixings among $\mathrm{CP}$-even and $\mathrm{CP}$-odd Higgs bosons.

It is of great interest to examine the possibility of studying these Higgs bosons in detail in CP non-invariant theories. A two-photon collision option [5] of the future linear $e^{+} e^{-}$colliders offers one of the ideal places to look for such Higgs signals [6]. There, neutral Higgs bosons can be produced via loop diagrams of charged particles. If the Higgs boson is lighter than about $140 \mathrm{GeV}$, its two-photon decay width can be measured accurately by looking for its main decay mode, which is usually the $b \bar{b}$ mode [7]. When the Higgs boson is heavy, the processes $\gamma \gamma \rightarrow W^{+} W^{-}, Z Z$ and $t \bar{t}$ can be useful to detect its signal [8]. In theories with weakly coupled Higgs sector, such as the MSSM, heavier Higgs bosons have suppressed branching fractions to the $W W / Z Z$ modes [9]. Furthermore, CP-odd Higgs bosons do not have the $Z Z$ decay modes in the tree level. On the other hand, the $t \bar{t}$ decay mode can be significant irrespective of the CP property of the Higgs boson $\phi$. In this case, it is expected that the heavy Higgs boson contributes to the process $\gamma \gamma \rightarrow t \bar{t}$ significantly around its mass pole.

The $s$-channel resonant amplitude of $\gamma \gamma \rightarrow \phi^{(*)} \rightarrow t \bar{t}$ can interfere with the tree-level $t$ - and $u$-channel continuum amplitudes, if the resonant and the continuum amplitudes have comparable magnitudes near the resonance pole [10]. This often happens for heavy Higgs bosons where both the peak resonant amplitudes and their total decay widths are large enough to make the interference effects significant. In this article, we study the contribution of a heavy Higgs boson without definite $\mathrm{CP}$-parity to the process $\gamma \gamma \rightarrow t \bar{t}$ and describe an efficient method to determine its CP property completely by making use of the photon and $t \bar{t}$ polarizations; the procedure is crucially 
based upon the interference effects among various helicity amplitudes. We also demonstrate the feasibility of determining the heavy Higgs-boson contribution in the context of the MSSM.

The remainder of this article is organized as follows. In Sect. 2, the helicity amplitudes of the process $\gamma \gamma \rightarrow t \bar{t}$ are calculated with model-independent parametrizations of the couplings of the Higgs boson $\phi$ to a photon pair and a top-quark pair. In Sect. 3 we present all the observables constructed by use of photon polarizations and by measuring the helicities of the final top and anti-top quarks. Section 4 gives the description of the procedure to determine the CP property of the Higgs boson completely. In Sect. 5 we study the properties of polarized photon beams through the Compton laser backscattering. In Sect. 6 we demonstrate the complete determination of the CP property of the heaviest neutral Higgs boson in the MSSM. Finally, we give conclusions in Sect. 7.

\section{Helicity amplitudes}

In CP non-invariant theories, the lowest-dimensional interaction of the Higgs boson $\phi$ with a top-quark pair can be described in a model-independent way by the vertex:

$$
\mathcal{V}_{\phi t \bar{t}}=-i e \frac{m_{t}}{m_{W}}\left(S_{t}+i \gamma_{5} P_{t}\right)
$$

and the loop-induced interaction of the Higgs boson with a photon pair is parameterized in a model-independent form as follows:

$$
\mathcal{V}_{\gamma \gamma \phi}=\sqrt{s} \frac{\alpha}{4 \pi}\left\{S_{\gamma}(s)\left[\epsilon_{1} \cdot \epsilon_{2}-\frac{2}{s}\left(\epsilon_{1} \cdot k_{2}\right)\left(\epsilon_{2} \cdot k_{1}\right)\right]-P_{\gamma}(s) \frac{2}{s}\left\langle\epsilon_{1} \epsilon_{2} k_{1} k_{2}\right\rangle\right\}
$$

where the form factors depend on the c.m. energy squared $s$ of two colliding photons, $\epsilon_{1,2}$ stand for the wave vectors of the two photons, $k_{1,2}$ are the four-momenta of the two photons, and

$$
\left\langle\epsilon_{1} \epsilon_{2} k_{1} k_{2}\right\rangle=\epsilon_{\mu \nu \alpha \beta} \epsilon_{1}^{\mu} \epsilon_{2}^{\nu} k_{1}^{\alpha} k_{2}^{\beta},
$$

with $\epsilon_{0123}=1$. Since we are interested in the $s$-channel virtual (and real) Higgs-boson exchange, for the sake of consistency the $s$-dependence of the form factors $S_{\gamma}$ and $P_{\gamma}$ is exhibited explicitly in Eq. (2). We note that a simultaneous presence of $\left\{S_{\gamma}, P_{\gamma}\right\}$ or/and $\left\{S_{t}, P_{t}\right\}$ implies CP noninvariance of the theory.

In the two-photon c.m coordinate system with $\vec{k}_{1}$ along the positive $z$ direction and $\vec{k}_{2}$ along the negative $z$ direction, the wave vectors $\epsilon_{1,2}$ of two photons are given by

$$
\epsilon_{1}^{\mu}(\lambda)=\epsilon_{2}^{\mu *}(\lambda)=\frac{-\lambda}{\sqrt{2}}(0,1, i \lambda, 0)
$$


where $\lambda= \pm 1$ denote the right and left photon helicities, respectively. Using Eqs. (11), (2) and (14) one can derive the explicit forms of the helicity amplitudes for the process $\gamma \gamma \rightarrow t \bar{t}$ which consist of two parts; the s-channel Higgs-boson exchange and the tree-level continuum contributions:

\section{(i) Higgs-exchange contribution:}

$$
\mathcal{M}_{\phi}^{\left(\lambda_{1} \lambda_{2}: \sigma \bar{\sigma}\right)}=\frac{e \alpha m_{t}}{4 \pi m_{W}} \frac{s}{s-m_{\phi}^{2}+i m_{\phi} \Gamma_{\phi}}\left[S_{\gamma}(s)+i \lambda_{1} P_{\gamma}(s)\right]\left[\sigma \beta S_{t}-i P_{t}\right] \delta_{\lambda_{1}, \lambda_{2}} \delta_{\sigma \bar{\sigma}}
$$

\section{(ii) Tree-level continuum contribution:}

$$
\begin{aligned}
\mathcal{M}_{\text {cont }}^{\left(\lambda_{1} \lambda_{2}: \sigma \bar{\sigma}\right)} & =\frac{4 \pi \alpha Q_{t}^{2}}{1-\beta^{2} \cos ^{2} \Theta}\left\{\frac{4 m_{t}}{\sqrt{s}}\left(\lambda_{1}+\sigma \beta\right) \delta_{\lambda_{1} \lambda_{2}} \delta_{\sigma \bar{\sigma}}\right. \\
& \left.-\frac{4 m_{t}}{\sqrt{s}} \sigma \beta \sin ^{2} \Theta \delta_{\lambda_{1},-\lambda_{2}} \delta_{\sigma \bar{\sigma}}-2 \beta\left(\cos \Theta+\lambda_{1} \sigma\right) \sin \Theta \delta_{\lambda_{1},-\lambda_{2}} \delta_{\sigma,-\bar{\sigma}}\right\}
\end{aligned}
$$

where $\Theta$ is the scattering angle of the top quark with respect to the positive $z$ direction,

$\beta=\sqrt{1-4 m_{t}^{2} / s}, \lambda_{1}= \pm 1\left(\lambda_{2}= \pm 1\right)$ denote the right and left helicities of the incident photon with its three-momentum along the positive (negative) direction, respectively, in units of $\hbar$, and $\sigma= \pm 1(\bar{\sigma}= \pm 1)$ denote the right and left helicities of $t(\bar{t})$, respectively, in units of $\hbar / 2$. There exists the interference between the Higgs-boson and the continuum contributions for equal $t$ and $\bar{t}$ helicities which can be used to observe the CP property of the Higgs boson. On the other hand, the continuum contribution with opposite $t$ and $\bar{t}$ helicities dominates the $\gamma \gamma \rightarrow t \bar{t}$ events at high energies so that it is important to distinguish events with equal $t$ and $\bar{t}$ helicities from those with opposite $t$ and $\bar{t}$ helicities. With the above points in mind, we concentrate on the helicity amplitudes of equal $t$ and $\bar{t}$ helicities in the present work.

When the helicities of the top and anti-top quarks are equal, i.e. $\sigma=\bar{\sigma}$, the helicity amplitudes for the process $\gamma \gamma \rightarrow t \bar{t}$ can be rewritten in the following simple form

$$
\begin{aligned}
\mathcal{M}^{(\lambda \lambda: \sigma \sigma)} & =A_{\text {cont }}(s)(\lambda+\sigma \beta)+A_{\phi}(s)\left[S_{\gamma}+i \lambda P_{\gamma}\right]\left[\sigma \beta S_{t}-i P_{t}\right] \\
\mathcal{M}^{(\lambda,-\lambda: \sigma \sigma)} & =-A_{\text {cont }}(s) \sigma \beta \sin ^{2} \Theta
\end{aligned}
$$

where for the sake of brevity the explicit $s$-dependence of the form factors $S_{\gamma}$ and $P_{\gamma}$ is not denoted and two $s$-dependent functions $A_{\text {cont }}(s)$ and $A_{\phi}(s)$ are introduced:

$$
A_{\mathrm{cont}}(s)=\frac{16 \pi \alpha Q_{t}^{2} m_{t}}{\sqrt{s}\left(1-\beta^{2} \cos ^{2} \Theta\right)}, \quad A_{\phi}(s)=\frac{e \alpha}{4 \pi} \frac{m_{t}}{m_{W}} D_{\phi}(s) .
$$


Here $D_{\phi}(s)$ is the $s$-channel propagator of the Higgs boson

$$
D_{\phi}(s)=\frac{s}{s-m_{\phi}^{2}+i m_{\phi} \Gamma_{\phi}} .
$$

A few important comments concerning the helicity amplitudes (7) are in order:

- Only the amplitudes with equal photon helicities have contributions from the Higgs-boson exchange.

- The spin-0 Higgs-boson contributions are independent of the scattering angle $\Theta$.

- The $s$-dependent form factors $S_{\gamma}$ and $P_{\gamma}$ as well as $A_{\phi}$ are in general complex while the other form factors are real in the leading order.

- The continuum part is $\mathrm{CP}$-preserving while the Higgs-exchange part can be $\mathrm{CP}$-violating if the 'scalar' (CP-even) form factors, $S_{\gamma}$ and $S_{t}$, and 'pseudoscalar' (CP-odd) form factors, $P_{\gamma}$ and $P_{t}$, are present simultaneously.

\section{Polarized cross sections}

\subsection{Equal photon helicities and top-quark helicities}

In this section, we investigate what physics information on the Higgs-boson contribution can be extracted from the cross section with equal photon and top-quark helicities. First of all, we can construct four independent squared amplitudes:

$$
\begin{aligned}
\left|\mathcal{M}^{(++:++)}\right|^{2} & =\overline{|\mathcal{M}|_{0}^{2}}\left[1+\mathcal{A}_{0}+\mathcal{A}_{1}-(1+\beta)\left(\mathcal{A}_{2}-\mathcal{A}_{3}\right)\right] \\
\left|\mathcal{M}^{(--:--)}\right|^{2} & =\overline{|\mathcal{M}|_{0}^{2}}\left[1+\mathcal{A}_{0}-\mathcal{A}_{1}+(1+\beta)\left(\mathcal{A}_{2}-\mathcal{A}_{3}\right)\right] \\
\left|\mathcal{M}^{(++:--)}\right|^{2} & =\overline{|\mathcal{M}|_{0}^{2}}\left[1-\mathcal{A}_{0}+\mathcal{A}_{1}+(1-\beta)\left(\mathcal{A}_{2}+\mathcal{A}_{3}\right)\right] \\
\left|\mathcal{M}^{(--:++)}\right|^{2} & =\overline{|\mathcal{M}|_{0}^{2}}\left[1-\mathcal{A}_{0}-\mathcal{A}_{1}-(1-\beta)\left(\mathcal{A}_{2}+\mathcal{A}_{3}\right)\right]
\end{aligned}
$$

where $\overline{|\mathcal{M}|_{0}^{2}}$ is the unpolarized squared amplitude, i.e. the average

$$
\overline{|\mathcal{M}|_{0}^{2}}=\frac{1}{4}\left[\left|\mathcal{M}^{(++:++)}\right|^{2}+\left|\mathcal{M}^{(--:--)}\right|^{2}+\left|\mathcal{M}^{(++:--)}\right|^{2}+\left|\mathcal{M}^{(--:++)}\right|^{2}\right]
$$

The explicit form (17) of the helicity amplitudes then leads to the following expressions for $\overline{|\mathcal{M}|_{0}^{2}}$ and the quantities $\mathcal{A}_{i}(i=0,1,2,3)$ :

$$
\overline{|\mathcal{M}|_{0}^{2}}=\left(1+\beta^{2}\right) A_{\text {cont }}^{2}+\left(\beta^{2} S_{t}^{2}+P_{t}^{2}\right)\left(\left|S_{\gamma}\right|^{2}+\left|P_{\gamma}\right|^{2}\right)\left|A_{\phi}\right|^{2}
$$




$$
\begin{gathered}
+2 A_{\text {cont }}\left[\beta^{2} S_{t} \mathcal{R}\left(A_{\phi} S_{\gamma}\right)+P_{t} \mathcal{R}\left(A_{\phi} P_{\gamma}\right)\right], \\
\mathcal{A}_{0}=2 \beta A_{\text {cont }}\left\{A_{\text {cont }}+\left[S_{t} \mathcal{R}\left(A_{\phi} S_{\gamma}\right)+P_{t} \mathcal{R}\left(A_{\phi} P_{\gamma}\right)\right]\right\} / \overline{|\mathcal{M}|_{0}^{2}}, \\
\mathcal{A}_{1}=2\left|A_{\phi}\right|^{2}\left\{\left(\beta^{2} S_{t}^{2}+P_{t}^{2}\right) \mathcal{I}\left(S_{\gamma} P_{\gamma}^{*}\right)\right\} / \overline{|\mathcal{M}|_{0}^{2}}, \\
\mathcal{A}_{2}=2 \beta A_{\text {cont }}\left\{S_{t} \mathcal{I}\left(A_{\phi} P_{\gamma}\right)\right\} / \overline{|\mathcal{M}|_{0}^{2}}, \\
\mathcal{A}_{3}=2 A_{\text {cont }}\left\{P_{t} \mathcal{I}\left(A_{\phi} S_{\gamma}\right)\right\} / \overline{|\mathcal{M}|_{0}^{2}},
\end{gathered}
$$

where $\overline{|\mathcal{M}|_{0}^{2}}$ and $\mathcal{A}_{0}$ are $\mathrm{CP}$-even, but the three asymmetries $\mathcal{A}_{1,2,3}$ are CP-odd, that is to say, they can be non-vanishing only in $\mathrm{CP}$ non-invariant theories. More explicitly, we can exploit the $\mathrm{CP}-$ odd combinations

$$
\begin{aligned}
& \sum_{\lambda} \lambda\left\{\left|\mathcal{M}^{(++: \lambda \lambda)}\right|^{2}+\left|\mathcal{M}^{(--: \lambda \lambda)}\right|^{2}\right\} / 4 \mid \overline{\left.\mathcal{M}\right|_{0} ^{2}}=-\mathcal{A}_{2}+\beta \mathcal{A}_{3} \\
& \sum_{\sigma} \sigma\left\{\left|\mathcal{M}^{(\sigma \sigma:++)}\right|^{2}+\left|\mathcal{M}^{(\sigma \sigma:--)}\right|^{2}\right\} / 4 \overline{|\mathcal{M}|_{0}^{2}}=\mathcal{A}_{1}-\beta \mathcal{A}_{2}+\mathcal{A}_{3},
\end{aligned}
$$

in extracting the $\mathrm{CP}$-odd asymmetries $\mathcal{A}_{1,2,3}$. It is however clear that we need to exploit more observables to determine all the form factors, $S_{\gamma}, S_{t}, P_{\gamma}$ and $P_{t}$ completely. This can be done by using linear photon polarization as well as circular photon polarization as shown in the next section.

\subsection{Two photon spin correlations}

Taking into account the general polarization configuration of two photon beams and taking the sum over the final polarization configuration with equal $t$ and $\bar{t}$ helicities, we obtain the polarized squared amplitude as

$$
\begin{aligned}
& \overline{|\mathcal{M}|^{2}=\overline{\mathcal{M}}_{0}^{2}}\left\{\left(1+\zeta_{2} \tilde{\zeta}_{2}\right)+\mathcal{B}_{1}\left(\zeta_{2}+\tilde{\zeta}_{2}\right)+\mathcal{B}_{2}\left(\zeta_{1} \tilde{\zeta}_{3}+\zeta_{3} \tilde{\zeta}_{1}\right)-\mathcal{B}_{3}\left(\zeta_{1} \tilde{\zeta}_{1}-\zeta_{3} \tilde{\zeta}_{3}\right)\right. \\
&+\sin ^{2} \Theta[-\mathcal{C}_{0}\left(\zeta_{2} \tilde{\zeta}_{2}-\zeta_{3} \tilde{\zeta}_{3}\right)+\mathcal{C}_{1}\left(\zeta_{1}+\tilde{\zeta}_{1}\right)+\mathcal{C}_{2}\left(\zeta_{3}+\tilde{\zeta}_{3}\right) \\
&\left.\left.+\mathcal{C}_{3}\left(\zeta_{1} \tilde{\zeta}_{2}+\zeta_{2} \tilde{\zeta}_{1}\right)+\mathcal{C}_{4}\left(\zeta_{2} \tilde{\zeta}_{3}+\zeta_{3} \tilde{\zeta}_{2}\right)\right]\right\}
\end{aligned}
$$

where the newly-introduced unpolarized squared amplitude ${\overline{|\mathcal{M}|_{0}^{2}}}^{\prime}$ is given by

$$
\overline{|\mathcal{M}|_{0}^{2}}=\overline{|\mathcal{M}|_{0}^{2}}+\beta^{2} \sin ^{4} \Theta A_{\text {cont }}^{2}
$$

The second term above comes from the continuum contributions with opposite photon helicities. The parameters $\left\{\zeta_{i}\right\}$ and $\left\{\tilde{\zeta}_{i}\right\}(i=1,2,3)$ are the Stokes parameters of two photon beams, 
respectively, which should be initially prepared. In Sect. 5 we will give a brief description of generating energetic photon beams and controlling their polarizations through the Compton laser backscattering off the electron or positron beams.

The observables $\mathcal{B}_{i}(i=1,2,3)$ in Eq. (14) are due to the interference of the continuum and Higgs-boson parts in the helicity amplitudes $\mathcal{M}^{(\lambda \lambda: \sigma \sigma)}$ of Eq. (7) and they are explicitly given by

$$
\begin{aligned}
\mathcal{B}_{1}= & \left(\mathcal{A}_{1}-\beta \mathcal{A}_{2}+\mathcal{A}_{3}\right) \overline{|\mathcal{M}|_{0}^{2}} / \overline{|\mathcal{M}|_{0}^{2}} \\
\mathcal{B}_{2}= & 2\left\{\left|A_{\phi}\right|^{2}\left(\beta^{2} S_{t}^{2}+P_{t}^{2}\right) \mathcal{R}\left(S_{\gamma} P_{\gamma}^{*}\right)+A_{\text {cont }}\left[\beta^{2} S_{t} \mathcal{R}\left(A_{\phi} P_{\gamma}\right)+P_{t} \mathcal{R}\left(A_{\phi} S_{\gamma}\right)\right]\right\} / \overline{|\mathcal{M}|_{0}^{2}} \\
\mathcal{B}_{3}= & \left\{\left(-1+\beta^{2}-\beta^{2} \sin ^{4} \Theta\right) A_{\text {cont }}^{2}+\left(\beta^{2} S_{t}^{2}+P_{t}^{2}\right)\left(\left|S_{\gamma}\right|^{2}-\left|P_{\gamma}\right|^{2}\right)\left|A_{\phi}\right|^{2}\right. \\
& \left.+2 A_{\text {cont }}\left[\beta^{2} S_{t} \mathcal{R}\left(A_{\phi} S_{\gamma}\right)-P_{t} \mathcal{R}\left(A_{\phi} P_{\gamma}\right)\right]\right\} / \overline{|\mathcal{M}|_{0}^{2}} .
\end{aligned}
$$

The observable $\mathcal{B}_{3}$ is $\mathrm{CP}$-even, while the other two observables $\mathcal{B}_{1,2}$ are $\mathrm{CP}-$ odd. We note that the latter $\mathrm{CP}$-odd observable $\mathcal{B}_{2}$ corresponds to the so-called $\mathrm{T}$-odd triple product of one photon momentum and two photon polarization vectors.

On the other hand, the five additional observables $\mathcal{C}_{i}(i=0$ to 4$)$ are due to the interference between the helicity amplitudes with equal photon helicities and those with opposite photon helicities and they are explicitly given by

$$
\begin{aligned}
& \mathcal{C}_{0}=2 \beta^{2} \sin ^{2} \Theta A_{\text {cont }}^{2} /{\overline{|\mathcal{M}|_{0}^{2}}}^{\prime}, \\
& \mathcal{C}_{1}=2 \beta^{2} A_{\text {cont }}\left\{S_{t} \mathcal{R}\left(A_{\phi} P_{\gamma}\right)\right\} /{\overline{|\mathcal{M}|_{0}^{2}}}^{\prime}, \\
& \mathcal{C}_{2}=2 \beta^{2} A_{\text {cont }}\left\{A_{\text {cont }}+S_{t} \mathcal{R}\left(A_{\phi} S_{\gamma}\right)\right\} /{\overline{|\mathcal{M}|_{0}^{2}}}^{\prime}, \\
& \mathcal{C}_{3}=2 \beta^{2} A_{\text {cont }}\left\{S_{t} \mathcal{I}\left(A_{\phi} S_{\gamma}\right)\right\} /{\overline{|\mathcal{M}|_{0}^{2}}}^{\prime}, \\
& \mathcal{C}_{4}=-2 \beta^{2} A_{\text {cont }}\left\{S_{t} \mathcal{I}\left(A_{\phi} P_{\gamma}\right)\right\} /{\overline{|\mathcal{M}|_{0}^{2}}}^{\prime} .
\end{aligned}
$$

Among these polarization asymmetries, the observables $\mathcal{C}_{0,2,3}$ are $\mathrm{CP}$-even, while the observables $\mathcal{C}_{1,4}$ are $\mathrm{CP}-$ odd.

\subsection{Top and anti-top quark polarizations}

At asymptotically high energies the chirality conservation of the gauge-interactions leads to the dominance of $t \bar{t}$-pair production with opposite helicity in the continuum amplitudes. However, near the threshold, there is also substantial production of $t \bar{t}$-pairs of the same helicity. The $t \bar{t}$ states with the same helicity transform to each other under CP, so any asymmetry in their 
production rates can provide a useful tool for studying $\mathrm{CP}$ violation.

Along with the initial two-photon polarizations we consider the final polarization configuration with equal $t \bar{t}$ helicities to construct the polarization asymmetry

$$
\Delta=\frac{\overline{|\mathcal{M}|^{2}}(\sigma=\bar{\sigma}=+)-\overline{|\mathcal{M}|^{2}}(\sigma=\bar{\sigma}=-)}{\mid \overline{\left.\mathcal{M}\right|_{0} ^{2}}} .
$$

Depending on the photon spin-spin correlations, the observable $\Delta$ is decomposed as follows:

$$
\begin{aligned}
\Delta= & \mathcal{D}_{1}\left(1+\zeta_{2} \tilde{\zeta}_{2}\right)+\mathcal{D}_{2}\left(\zeta_{2}+\tilde{\zeta}_{2}\right)+\mathcal{D}_{3}\left(\zeta_{1} \tilde{\zeta}_{3}+\zeta_{3} \tilde{\zeta}_{1}\right)-\mathcal{D}_{4}\left(\zeta_{1} \tilde{\zeta}_{1}-\zeta_{3} \tilde{\zeta}_{3}\right) \\
& +\sin ^{2} \Theta\left[\mathcal{E}_{1}\left(\zeta_{1}+\tilde{\zeta}_{1}\right)+\mathcal{E}_{2}\left(\zeta_{3}+\tilde{\zeta}_{3}\right)+\mathcal{E}_{3}\left(\zeta_{1} \tilde{\zeta}_{2}+\zeta_{2} \tilde{\zeta}_{1}\right)+\mathcal{E}_{4}\left(\zeta_{2} \tilde{\zeta}_{3}+\zeta_{3} \tilde{\zeta}_{2}\right)\right]
\end{aligned}
$$

The observables $\mathcal{D}_{i}(i=1$ to 4$)$ are due to the interference of the continuum and Higgs-boson parts in the helicity amplitudes $\mathcal{M}^{(\lambda \lambda: \sigma \sigma)}$ and they are explicitly given by

$$
\begin{aligned}
& \mathcal{D}_{1}=2 \beta A_{\text {cont }}\left\{-S_{t} \mathcal{I}\left(A_{\phi} P_{\gamma}\right)+P_{t} \mathcal{I}\left(A_{\phi} S_{\gamma}\right)\right\} /{\overline{|\mathcal{M}|_{0}^{2}}}^{\prime}, \\
& \mathcal{D}_{2}=2 \beta A_{\text {cont }}\left\{A_{\text {cont }}+\left[S_{t} \mathcal{R}\left(A_{\phi} S_{\gamma}\right)+P_{t} \mathcal{R}\left(A_{\phi} P_{\gamma}\right)\right]\right\} / \overline{|\mathcal{M}|_{0}^{2}}, \\
& \mathcal{D}_{3}=2 \beta A_{\text {cont }}\left\{-S_{t} \mathcal{I}\left(A_{\phi} S_{\gamma}\right)+P_{t} \mathcal{I}\left(A_{\phi} P_{\gamma}\right)\right\} /{\overline{|\mathcal{M}|_{0}^{2}}}^{\prime}, \\
& \mathcal{D}_{4}=2 \beta A_{\text {cont }}\left\{S_{t} \mathcal{I}\left(A_{\phi} P_{\gamma}\right)+P_{t} \mathcal{I}\left(A_{\phi} S_{\gamma}\right)\right\} / \overline{|\mathcal{M}|_{0}^{\prime}}
\end{aligned}
$$

The observables $\mathcal{D}_{1,4}$ are $\mathrm{CP}$-odd and the observables $\mathcal{D}_{2,3}$ are $\mathrm{CP}$-even.

On the other hand, the four additional observables $\mathcal{E}_{i}(i=1$ to 4 ) come from the interference between the helicity amplitudes with equal photon helicities and those with opposite photon helicities. Their explicit forms are

$$
\begin{aligned}
& \mathcal{E}_{1}=2 \beta A_{\text {cont }}\left\{P_{t} \mathcal{I}\left(A_{\phi} P_{\gamma}\right)\right\} /{\overline{|\mathcal{M}|_{0}^{2}}}^{\prime}, \\
& \mathcal{E}_{2}=2 \beta A_{\text {cont }}\left\{P_{t} \mathcal{I}\left(A_{\phi} S_{\gamma}\right)\right\} / \overline{|\mathcal{M}|_{0}^{2}}, \\
& \mathcal{E}_{3}=-2 \beta A_{\text {cont }}\left\{P_{t} \mathcal{R}\left(A_{\phi} S_{\gamma}\right)\right\} /{\overline{|\mathcal{M}|_{0}^{2}}}^{\prime}, \\
& \mathcal{E}_{4}=2 \beta A_{\text {cont }}\left\{A_{\text {cont }}+P_{t} \mathcal{R}\left(A_{\phi} P_{\gamma}\right)\right\} /{\overline{|\mathcal{M}|_{0}^{2}}}^{\prime} .
\end{aligned}
$$

The observables $\mathcal{E}_{1,4}$ are $\mathrm{CP}$-even and the observables $\mathcal{E}_{2,3}$ are $\mathrm{CP}$-odd. 


\section{Complete measurements of the Higgs-boson CP prop- erty}

In order to completely determine the CP parity of the Higgs boson we need to measure the following six quantities (see Eq. (5)):

$$
\left\{m_{\phi}, \Gamma_{\phi}, S_{\gamma}, P_{\gamma}, S_{t}, P_{t}\right\}
$$

among which the one-loop induced $\gamma \gamma \phi$ form factors $S_{\gamma}$ and $P_{\gamma}$ are in general complex while the others are real in the leading order. However, the helicity amplitudes are determined by helicitydependent multiplications of those quantities so that it is necessary to measure the following quantities:

$$
\begin{array}{llll}
S_{t} \mathcal{R}\left(A_{\phi} S_{\gamma}\right), & S_{t} \mathcal{R}\left(A_{\phi} P_{\gamma}\right), & S_{t} \mathcal{I}\left(A_{\phi} S_{\gamma}\right), & S_{t} \mathcal{I}\left(A_{\phi} P_{\gamma}\right) \\
P_{t} \mathcal{R}\left(A_{\phi} S_{\gamma}\right), & P_{t} \mathcal{R}\left(A_{\phi} P_{\gamma}\right), & P_{t} \mathcal{I}\left(A_{\phi} S_{\gamma}\right), & P_{t} \mathcal{I}\left(A_{\phi} P_{\gamma}\right)
\end{array}
$$

The above 8 quantities are not completely independent and satisfy, for example, the following relations at all $s$;

$$
\begin{aligned}
P_{t} \mathcal{R}\left(A_{\phi} S_{\gamma}\right) \cdot S_{t} \mathcal{R}\left(A_{\phi} P_{\gamma}\right) & =P_{t} \mathcal{R}\left(A_{\phi} P_{\gamma}\right) \cdot S_{t} \mathcal{R}\left(A_{\phi} S_{\gamma}\right) \\
P_{t} \mathcal{I}\left(A_{\phi} S_{\gamma}\right) \cdot S_{t} \mathcal{I}\left(A_{\phi} P_{\gamma}\right) & =P_{t} \mathcal{I}\left(A_{\phi} P_{\gamma}\right) \cdot S_{t} \mathcal{I}\left(A_{\phi} S_{\gamma}\right)
\end{aligned}
$$

On the other hand, in principle 22 observables are available as shown in the previous section; $\overline{|\mathcal{M}|_{0}^{2}}, \overline{|\mathcal{M}|_{0}^{2}}{ }^{\prime}, 4 \mathcal{A}$ 's, $3 \mathcal{B}$ 's, $5 \mathcal{C}$ 's, $4 \mathcal{D}^{\prime}$ s and $4 \mathcal{E}$ 's. Therefore, it is expected that the Higgs-boson parameters are completely determined and that they are over-constrained.

Assuming that each observable is measured with a reasonable efficiency, we provide a straightforward procedure to determine the quantities listed in Eq. (23):

(1) The first four quantities in Eq. (23) can be determined directly through four observables $\mathcal{C}_{i}(i=1$ to 4$)$ even without measuring top and anti-top helicities.

(2) The remaining four quantities in Eq. (23) can be determined directly through four observables $\mathcal{E}_{i}(i=1$ to 4$)$.

(3) The constraints of Eq. (24), the observables $\mathcal{D}_{i}(i=1$ to 4$)$, and also $\mathcal{A}_{i}(i=0$ to 3$)$ and $\mathcal{B}_{i}(i=1$ to 3$)$ can be used to test and improve the above measurements.

To recapitulate, the Higgs-exchange contribution can be completely determined by a judicious use of photon polarizations and $t \bar{t}$ helicity measurements. 


\section{$5 \quad$ Polarized high energy photon beams}

The observed cross section is a convoluted one of the parton-level cross section with a (polarized) $\gamma \gamma$ luminosity function describing the spread of the $\gamma \gamma$ collision energy. A detailed study of the possible luminosity and polarization distributions at future $\gamma \gamma$ colliders has been performed by the simulation program CAIN [11]. However, these quantities are strongly dependent on the machine design of the colliders. Thus we adopt an ideal situation of the beam conversion that the photon beam is generated by the tree-level formula of the Compton backward-scattering and that the effect of the finite scattering angle is negligible [12.

High energy colliding beams of polarized photons can be generated by Compton backscattering of polarized laser light on (polarized) electron/positron bunches of $e^{+} e^{-}$linear colliders ${ }^{\natural}$. The polarization transfer from the laser light to the high energy photons is described by three Stokes parameters $\zeta_{1,2,3} ; \zeta_{2}$ is the degree of circular polarization and $\left\{\zeta_{3}, \zeta_{1}\right\}$ the degree of linear polarization transverse and normal to the plane defined by the electron direction and the direction of the maximal linear polarization of the initial laser light. Explicitly, the Stokes parameters take the form [12]:

$$
\zeta_{1}=\frac{f_{3}(y)}{f_{0}(y)} P_{t} \sin 2 \kappa, \quad \zeta_{2}=-\frac{f_{2}(y)}{f_{0}(y)} P_{c}, \quad \zeta_{3}=\frac{f_{3}(y)}{f_{0}(y)} P_{t} \cos 2 \kappa,
$$

where $y$ is the energy fraction of the back-scattered photon with respect to the initial electron energy $E_{e},\left\{P_{c}, P_{t}\right\}$ are the degrees of circular and transverse polarization of the initial laser light, and $\kappa$ is the azimuthal angle between the directions of initial photon and its maximum linear polarization. Similar relations can be obtained for the Stokes parameters $\tilde{\zeta}$ of the opposite high energy photon beam by replacing $\left(P_{c}, P_{t}, \kappa\right)$ with $\left(\tilde{P}_{c}, \tilde{P}_{t},-\tilde{\kappa}\right)$. The functions $f_{0}, f_{2}$, and $f_{3}$ determining the photon energy spectrum and the Stokes parameters are given by

$$
\begin{aligned}
& f_{0}(y)=\frac{1}{1-y}+1-y-4 r(1-r), \\
& f_{2}(y)=(2 r-1)\left(\frac{1}{1-y}+1-y\right), \\
& f_{3}(y)=2 r^{2}
\end{aligned}
$$

with $r=y / x(1-y)$ and

$$
x=\frac{4 E_{e} \omega_{0}}{m_{e}^{2}} \approx 15.4\left(\frac{E_{e}}{\mathrm{TeV}}\right)\left(\frac{\omega_{0}}{\mathrm{eV}}\right)
$$

for the initial laser energy $\omega_{0}$. We note from Eq. (25) that the linear polarization of the high energy photon beam is proportional to $P_{t}$ whereas the circular polarization is proportional to $P_{c}$.

\footnotetext{
${ }^{a}$ In the present work the electron and positron beams are assumed to be unpolarized. It is however straightforward to take into account polarized electron and positron beams.
} 
Thus it is necessary to have both circularly and linearly polarized initial laser beams so as to measure all the polarization asymmetries $\mathcal{B}_{i}$ 's and $\mathcal{C}_{i}$ 's through the distribution (14).

After folding the luminosity spectra of two photon beams, the event rate of the process $\gamma \gamma \rightarrow t \bar{t}$ is given by

$$
\begin{aligned}
\frac{\mathrm{d}^{3} N}{\mathrm{~d} \tau \mathrm{d} \Phi \mathrm{d} \cos \Theta}= & \frac{\mathrm{d} L_{\gamma \gamma}}{\mathrm{d} \tau} \frac{\mathrm{d} \hat{\sigma}_{0}}{\mathrm{~d} \cos \Theta}\left\{1+\langle 22\rangle_{\tau} P_{c} \tilde{P}_{c}-\langle 02\rangle_{\tau}\left(P_{c}+\tilde{P}_{c}\right) \mathcal{B}_{1}\right. \\
& +\langle 33\rangle_{\tau} P_{t} \tilde{P}_{t}\left[\sin 2(\kappa-\tilde{\kappa}) \mathcal{B}_{2}+\cos 2(\kappa-\tilde{\kappa}) \mathcal{B}_{3}\right] \\
& -\sin ^{2} \Theta\left[\left(\langle 22\rangle_{\tau} P_{c} \tilde{P}_{c}-\langle 33\rangle_{\tau} P_{t} \tilde{P}_{t} \cos 2 \kappa \cos 2 \tilde{\kappa}\right) \mathcal{C}_{0}\right. \\
& -\langle 03\rangle_{\tau}\left(\left\{P_{t} \sin 2 \kappa-\tilde{P}_{t} \sin 2 \tilde{\kappa}\right\} \mathcal{C}_{1}+\left\{P_{t} \cos 2 \kappa+\tilde{P}_{t} \cos 2 \tilde{\kappa}\right\} \mathcal{C}_{2}\right) \\
& \left.\left.+\langle 23\rangle_{\tau}\left(\left\{P_{t} \tilde{P}_{c} \sin 2 \kappa-\tilde{P}_{t} P_{c} \sin 2 \tilde{\kappa}\right\} \mathcal{C}_{3}+\left\{P_{t} \tilde{P}_{c} \cos 2 \kappa+\tilde{P}_{t} P_{c} \cos 2 \tilde{\kappa}\right\} \mathcal{C}_{4}\right)\right]\right\},
\end{aligned}
$$

where $\Phi$ is an azimuthal angle to be identifiable with $\kappa$, the function $\mathrm{d} L_{\gamma \gamma} / \mathrm{d} \tau$ is the two-photon luminosity function depending on the details such as the $e-\gamma$ conversion factor and the shape of the electron/positron bunches [12], and $\tau \equiv s / s_{e e}$. The differential cross-section is then given by

$$
\frac{\mathrm{d} \hat{\sigma}_{0}}{\mathrm{~d} \cos \Theta}=\frac{\beta}{32 \pi s}{\overline{|\mathcal{M}|_{0}^{2}}}^{\prime} .
$$

The correlation ratios $\langle i j\rangle_{\tau}(i, j=0$ to 3$)$ are defined as

$$
\langle i j\rangle_{\tau} \equiv \frac{\left\langle f_{i} * f_{j}\right\rangle_{\tau}}{\left\langle f_{0} * f_{0}\right\rangle_{\tau}}
$$

where the correlation function $\left\langle f_{i} * f_{j}\right\rangle_{\tau}$ is given by the integrated function

$$
\left\langle f_{i} * f_{j}\right\rangle_{\tau}=\int_{\tau / y_{\max }}^{y_{\max }} \frac{\mathrm{d} y}{y} f_{i}(y) f_{j}(\tau / y)
$$

with $y_{\max }=x /(1+x)$. The difference $\kappa-\tilde{\kappa}$ of two azimuthal angles $\kappa$ and $\tilde{\kappa}$ is independent of the azimuthal angle $\Phi$ while each of them is linearly dependent on the angle $\Phi$. This implies that the measurements of the observables $\mathcal{C}_{i}$ require the reconstruction of the scattering plane, which can be done statistically.

Figure 1 shows the unpolarized correlation function $\left\langle f_{0} * f_{0}\right\rangle_{\tau}$ and the five correlation ratios $\left\{\langle 02\rangle_{\tau},\langle 03\rangle_{\tau},\langle 22\rangle_{\tau},\langle 23\rangle_{\tau},\langle 33\rangle_{\tau}\right\}$ appearing in Eq. (28) for $x=0.5$ (solid line), 1.0 (dashed line), and 4.83 (dotted line). For a larger value of $x$, the correlation function $\left\langle f_{0} * f_{0}\right\rangle_{\tau}$, to which $\mathrm{d} L_{\gamma \gamma} / \mathrm{d} \tau$ is proportional in the ideal situation of the beam conversion, becomes more flat and the maximally-obtainable photon energy fraction becomes closer to the electron beam energy. Exploiting this feature appropriately could facilitate Higgs-boson searches at the photon collider. 

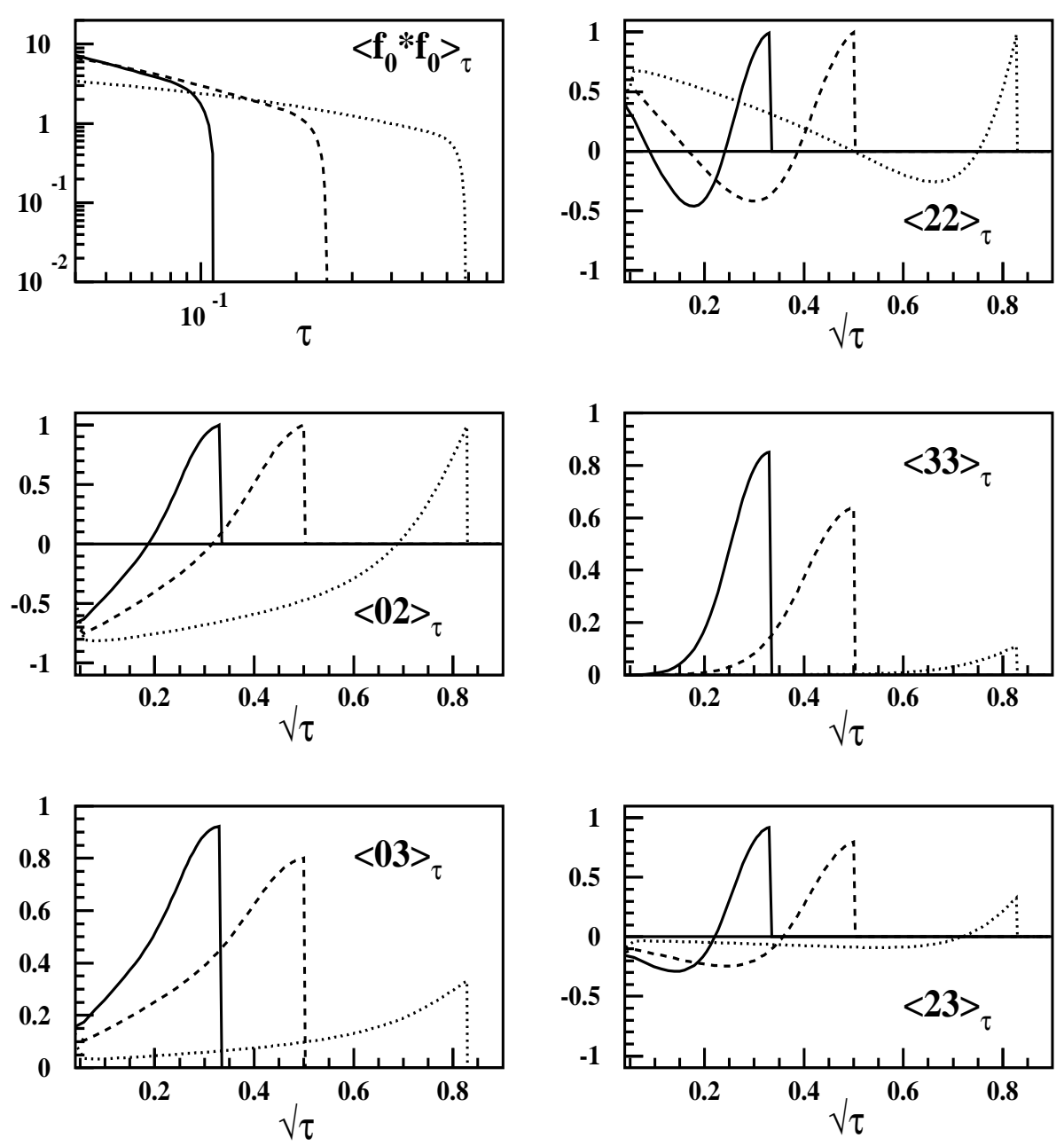

Figure 1: The unpolarized correlation function $\left\langle f_{0} * f_{0}\right\rangle_{\tau}$ and the five ratios $\langle i j\rangle_{\tau}$ of the correlation functions for three $x$ values; $x=0.5$ (solid line), 1.0 (dashed line), and 4.83 (dotted line). 
The five figures for the correlation ratios clearly show that the maximal sensitivity to each polarization asymmetry of $\mathcal{B}_{i}$ and $\mathcal{C}_{i}$ can be acquired near the maximal value of $\tau=y_{\max }^{2}$. Therefore, once the Higgs-boson mass is known, one can obtain the maximal sensitivities by tuning the initial electron energy to be

$$
E_{e}=\left(\frac{1+x}{2 x}\right) m_{\phi}
$$

On the other hand, the ratios $\langle 33\rangle_{\tau},\langle 03\rangle_{\tau}$ and $\langle 23\rangle_{\tau}$ are larger for a smaller value of $x$ and for a given $x$ the maximum value of the ratio $\langle 33\rangle_{\tau}$ is given by

$$
\langle 33\rangle_{\tau_{\max }}=\left[\frac{2(1+x)}{1+(1+x)^{2}}\right]^{2}
$$

Consequently, it is necessary to take a small $x$ and a high $E_{e}$ by changing the laser beam energy $\omega_{0}$ so as to acquire the highest sensitivity to $\mathrm{CP}$ violation in the neutral Higgs sector.

\section{An example in the MSSM}

The MSSM Higgs sector constitutes a typical two-Higgs-doublet model in which CP violation can be induced at the loop level from the stop and sbottom sectors through the complex trilinear parameters $A_{t, b}$ and the higgsino mass parameter $\mu$ [3, 4]. Although there exist three neutral Higgs bosons, we consider only the heaviest Higgs boson so as to estimate the unpolarized partonlevel cross section and the polarization asymmetries, which will allow us to completely determine the CP property of the Higgs boson following the procedure described in Sect. 4 .

Table 1: The mass and width $\left\{m_{\phi}, \Gamma_{\phi}\right\}$ and the four form factors $\left\{S_{\gamma}, P_{\gamma}, S_{t}, P_{t}\right\}$ of the heaviest MSSM Higgs boson for the parameter set (34) and $\tan \beta=3,10$.

\begin{tabular}{|c||c|c|c|c|c|c|}
\hline $\tan \beta$ & $m_{\phi}[\mathrm{GeV}]$ & $\Gamma_{\phi}[\mathrm{GeV}]$ & $S_{\gamma}$ & $P_{\gamma}$ & $S_{t}$ & $P_{t}$ \\
\hline \hline 3 & 500 & 1.9 & $-1.3-1.2 i$ & $-0.51+1.1 i$ & 0.33 & 0.15 \\
\hline 10 & 500 & 1.1 & $-0.39-0.35 i$ & $-0.06+0.14 i$ & 0.11 & 0.02 \\
\hline
\end{tabular}

In the MSSM the form factors $S_{\gamma}$ and $P_{\gamma}$, which describe the coupling of the Higgs boson to two photons, have the loop contributions from the bottom and top quarks, the charged Higgs boson, the $W$ boson, and the lighter top and bottom squarks as well as other charged particles such as charginos and heavier top and bottom squarks. The contributions from the charginos and heavier top and bottom squarks are neglected in the present work by taking them to be heavy. For our numerical example based on the work [4], we assume a universal trilinear parameter $A=A_{t}=A_{b}$ and take the physically-invariant phase $\Phi_{A \mu}=\Phi_{A}+\Phi_{\mu}$ to be $\pi / 2$, leading to the 
(almost) maximal CP violation. Then, we take for the remaining dimensionful parameters the parameter set:

$$
|A|=1.0 \mathrm{TeV}, \quad|\mu|=2.0 \mathrm{TeV}, \quad M_{\tilde{Q}_{L}}^{2}=M_{\tilde{t}_{R}}^{2}=M_{\tilde{b}_{R}}^{2}=(0.5 \mathrm{TeV})^{2}, \quad M_{H^{ \pm}}=0.5 \mathrm{TeV}
$$

where $M_{\tilde{Q}_{L}}^{2}, M_{\tilde{t}_{R}}^{2}$, and $M_{\tilde{b}_{R}}^{2}$ are the soft SUSY breaking top/bottom squark masses squared and $M_{H^{ \pm}}$is the charged Higgs boson mass. In addition, we take two values of $\tan \beta, \tan \beta=3$ and 10 , so as to obtain a crude estimate of the dependence of the form factors on $\tan \beta$. For the above MSSM parameters, the mass, the width, and the four form factors, $\left\{m_{\phi}, \Gamma_{\phi}, S_{\gamma}, P_{\gamma}, S_{t}, P_{t}\right\}$, of the heaviest MSSM Higgs boson on the mass pole $\sqrt{s}=m_{\phi}$ are presented in Table 1. Several comments on our results are in order:

- The Higgs-boson width is reduced for large $\tan \beta$. This is due to the suppression of the dominant partial decay width $\Gamma(\phi \rightarrow t \bar{t})$.

- The absolute values of all the form factors are very small for large tan $\beta$, leading to a strong suppression of the Higgs-boson contribution to the process $\gamma \gamma \rightarrow t \bar{t}$. In particular, the 'pseudoscalar' couplings, $P_{t}$ and $P_{\gamma}$, are very small, which implies an (almost) CP-even heavy Higgs boson.

One natural consequence from the $\tan \beta$ dependence of the form factors is that all the $\mathrm{CP}-$ odd polarization asymmetries are strongly suppressed for large $\tan \beta$.

We integrate the polarized distributions over the angular variables so as to obtain the unpolarized parton-level cross section $\hat{\sigma}_{0}$ and the averaged polarization asymmetries:

$$
\overline{\mathcal{A}_{i}} \equiv\left\langle\mathcal{A}_{i}\right\rangle, \quad \overline{\mathcal{B}_{i}} \equiv\left\langle\mathcal{B}_{i}\right\rangle^{\prime}, \quad \overline{\mathcal{C}_{i}} \equiv\left\langle\mathcal{C}_{i} \sin ^{2} \Theta\right\rangle^{\prime}, \quad \overline{\mathcal{D}_{i}} \equiv\left\langle\mathcal{D}_{i}\right\rangle^{\prime}, \quad \overline{\mathcal{E}_{i}} \equiv\left\langle\mathcal{E}_{i} \sin ^{2} \Theta\right\rangle^{\prime}
$$

where $\langle X\rangle\left(\langle X\rangle^{\prime}\right)$ denotes the average over the distribution $\overline{|\mathcal{M}|_{0}^{2}}\left(\overline{|\mathcal{M}|_{0}^{2}}\right)$. We do not present the polarized distributions folded with the photon luminosity spectrum explicitly because those distributions can be obtained in a rather straightforward way from the parton-level cross sections. We do not take into account the QCD radiative corrections either, but for the details we refer to Ref. [13].

Table 2 shows the parton-level unpolarized cross section $\hat{\sigma}_{0}$ and polarization asymmetries $\overline{\mathcal{A}}$ 's, which are constructed with equal photon helicities and equal $t \bar{t}$ helicities, in the MSSM parameter set (34) for the CP phase $\Phi_{A \mu}=\pi / 2$. Each sign \pm in the square brackets is for the $\mathrm{CP}$-parity of the observable. Note that the CP-odd observables $\overline{\mathcal{A}_{1}}, \overline{\mathcal{A}_{2}}$ and $\overline{\mathcal{A}_{3}}$ are significantly suppressed for $\tan \beta=10$ as expected.

In Tables 3 and 4 , we show the polarization asymmetries $\left\{\overline{\mathcal{B}_{i}}, \overline{\mathcal{C}_{i}}\right\}$ constructed with general two-photon spin correlations, and the polarization asymmetries $\overline{\mathcal{D}}$ 's and $\overline{\mathcal{E}}$ 's with general twophoton spin correlations and equal $t$ and $\bar{t}$ helicities, in the MSSM parameter set (34). The 
Table 2: $\quad$ The parton-level cross section and polarization asymmetries $\overline{\mathcal{A}}$ 's, which are constructed with equal photon and top-pair helicities, in the MSSM parameter set (34) for the CP phase $\Phi_{A \mu}=\pi / 2$. Each sign \pm in the square brackets is for the CP-parity of the observable.

\begin{tabular}{|c||c|c|c|c|c|}
\hline $\tan \beta$ & $\hat{\sigma}_{0}[+]$ & $\overline{\mathcal{A}_{0}}[+]$ & $\overline{\mathcal{A}_{1}}[-]$ & $\overline{\mathcal{A}_{2}}[-]$ & $\overline{\mathcal{A}_{3}}[-]$ \\
\hline \hline 3 & $0.88 \mathrm{pb}$ & 0.45 & 0.13 & -0.17 & 0.26 \\
\hline 10 & $0.62 \mathrm{pb}$ & 0.91 & 0.00 & -0.02 & 0.03 \\
\hline
\end{tabular}

Table 3: The values of the polarization asymmetries $\left\{\overline{\mathcal{B}_{i}}\right.$ and $\left.\overline{\mathcal{C}_{i}}\right\}$, which are constructed with general two-photon spin correlations, in the MSSM parameter set (34) for the CP phase $\Phi=\pi / 2$. Each sign \pm in the square brackets is for the CP-parity of the observable.

\begin{tabular}{|c||c|c|c||c|c|c|c|c|}
\hline $\tan \beta$ & $\overline{\mathcal{B}_{1}}[-]$ & $\overline{\mathcal{B}_{2}}[-]$ & $\overline{\mathcal{B}_{3}}[+]$ & $\overline{\mathcal{C}_{0}}[+]$ & $\overline{\mathcal{C}_{1}}[-]$ & $\overline{\mathcal{C}_{2}}[+]$ & $\overline{\mathcal{C}_{3}}[+]$ & $\overline{\mathcal{C}_{4}}[-]$ \\
\hline \hline 3 & 0.46 & -0.27 & -0.60 & 0.17 & 0.13 & 0.09 & 0.17 & 0.06 \\
\hline 10 & 0.03 & 0.00 & -0.47 & 0.24 & 0.01 & 0.30 & 0.04 & 0.00 \\
\hline
\end{tabular}

re-phasing-invariant phase $\Phi_{A \mu}$ is taken to be $\pi / 2$ and each signature \pm in the square brackets is for the $\mathrm{CP}$-parity of the observable as in Table 2. The $\mathrm{CP}$-odd polarization asymmetries are sizable for $\tan \beta=3$, but they are strongly suppressed for $\tan \beta=10$. In addition, the CP-even observables which are dependent only on the products $S_{\gamma} P_{\gamma}$ and $S_{t} P_{t}$ of the form factors are also suppressed for large $\tan \beta$. In particular, we note that the polarization asymmetry $\mathcal{E}_{1}$ is determined by only the CP-odd ('pseudoscalar') form factors $P_{\gamma}$ and $P_{t}$ so that its strong suppression implies an almost $\mathrm{CP}$-even Higgs boson.

Table 4: The polarization asymmetries $\overline{\mathcal{D}}$ 's and $\overline{\mathcal{E}}$ 's, which are constructed with general twophoton spin correlations and equal top-pair helicities, in the MSSM parameter set (34). The rephasing-invariant phase $\Phi_{A \mu}$ is taken to be $\pi / 2$ and only the interference between the continuum and the heaviest Higgs boson in the MSSM is taken into account. The signature \pm in the square brackets denotes the CP-parity of the corresponding observable.

\begin{tabular}{|c||c|c|c|c||c|c|c|c|}
\hline $\tan \beta$ & $\overline{\mathcal{D}_{1}}[-]$ & $\overline{\mathcal{D}_{2}}[+]$ & $\overline{\mathcal{D}_{3}}[+]$ & $\overline{\mathcal{D}_{4}}[-]$ & $\overline{\mathcal{E}_{1}}[+]$ & $\overline{\mathcal{E}_{2}}[-]$ & $\overline{\mathcal{E}_{3}}[-]$ & $\overline{\mathcal{E}_{4}}[+]$ \\
\hline \hline 3 & 0.32 & 0.41 & -0.45 & 0.03 & -0.04 & 0.10 & 0.09 & 0.40 \\
\hline 10 & 0.03 & 0.80 & -0.09 & 0.00 & -0.00 & 0.01 & 0.01 & 0.46 \\
\hline
\end{tabular}




\section{Conclusions}

In this article, we have studied the effects of a neutral Higgs boson without definite CP-parity in the process $\gamma \gamma \rightarrow t \bar{t}$ in a model-independent way.

We have found that the interference between the Higgs-boson-exchange and the continuum amplitudes with polarized photon beams and with the top and anti-top helicity measurements enables us to determine the CP property of a neutral Higgs boson completely even in CP noninvariant theories. We have classified the physical observables such as the unpolarized cross sections and polarization asymmetries depending on the polarization configuration of the initial two-photon beams and the helicity configuration of the final $t \bar{t}$ system. As an demonstration

of the procedure, the contribution of the heaviest neutral Higgs boson in the MSSM has been investigated quantitatively.

Certainly, the precision with which the Higgs-boson contribution is determined depends on the background processes as well as the efficiencies for controlling beam polarization and measuring the $t$ and $\bar{t}$ helicities. Nevertheless, the algorithm presented in the present work with more than 20 polarization observables will be helpful in determining the $s$-channel Higgs contribution to the process $\gamma \gamma \rightarrow t \bar{t}$ efficiently even in the CP non-invariant theories.

\section{Acknowledgements}

S.Y.C. wishes to acknowledge financial support of the 1997 Sughak program of the Korea Research Foundation.

\section{References}

[1] See, J.F. Gunion, H.E. Haber, G. Kane and S. Dawson, 'Higgs Hunter's Guide', AddisonWesley Publishing Company, (1990), and references therein; M. Spira and P.M. Zerwas, hep-ph/9803257.

[2] N. Ruis and V. Sanz, Nucl. Phys. B570, 155 (2000) and references therein.

[3] A. Pilaftsis, Phys. Lett. B435, 88 (1998), hep-ph/9805373; Phys. Rev. D58, 096010 (1998), hep-ph/9803297; A. Pilaftsis and C.E.M. Wagner, Nucl. Phys. B553, 3 (1999), hepph/9803297; D.A. Demir, Phys. Rev. D60, 055006 (1999), hep-ph/9901389; M. Carena, J. Ellis, A. Pilaftsis and C.E.M. Wagner, hep-ph/0003180.

[4] S.Y. Choi, M. Drees and J.S. Lee, hep-ph/0002287, (to appear in Phys. Lett. B).

[5] I.F. Ginzburg, G.L. Serbo and V.I. Telnov, Pis'ma Zh. Eksp. Teor. Fiz., 34 (1981) 514 [JETP Lett., 34 (1982) 491]; Nucl. Instrum. Methods, 205 (1083) 47. 
[6] J.F. Gunion and H.E. Haber, Phys. Rev. D 48, 5109 (1993); D.L. Borden, D.A. Bauer and D.O. Caldwell, ibid. D 48, 4018 (1993); I. Watanabe et al., KEK Report 97-17, KEK, March 1998; J.I. Illana, hep-ph/9912467 and references therein.

[7] T. Ohgaki, T. Takahashi and I. Watanabe, Phys. Rev., D56 (1997) 1723.

[8] G.V. Jikia, Nucl. Phys., B405 (1993) 24; M.S. Berger, Phys. Rev. D48 (1993) 5121; B. Grazadkowski and J.F. Gunion, Phys. Lett. B291 (1992) 361; M. Krämer, J. Kühn, M.L. Stong and P.M. Zerwas, Z. Phys. C64 (1994) 21; J.F. Gunion and J.G. Kelly, Phys. Lett. B333 (1994) 110; G.J. Gounaris and G.P. Tsirigoti, Phys. Rev. D 56, 3030 (1997); D 58, 059901 (1998) (E).

[9] A. Djouadi, J. Kalinowski and M. Spira, Com. Phys. Commun. Res. 108, 56 (1998).

[10] E. Asakawa, to be published in the proceedings of "4th International Workshop on Linear Colliders (LCWS99)", Sitges, Barcelona, Spain, 28 Apr. - 5 May, 1999; I. Watanabe, ibid.; E. Asakawa, J. Kamoshita, A. Sugamoto and I. Watanabe, hep-ph/9912373 (to appear in Eur. Phys. J. C).

[11] P. Chen, G. Horton-Smith, T. Ohgaki, A.W. Weidemann and K. Yokoya, Nucl. Instrum. Methods, A335 (1995) 107; T. Ohgaki and T. Takahashi, Nucl. Instrum. Methods, A373 (1996) 185; 'CAIN 2.1b', K. Yokoya, see the CAIN home page, http://www-acc-theory.kek.jp/members/cain/default.html.

[12] I.F. Ginzburg, G.L. Kotkin, S.L. Panfil, V.G. Serbo and V.I. Telnov, Nucl. Instrum. Methods, A219 (1984) 5; V.I. Telnov, Nucl. Instrum. Methods, A294 (1990) 72.

[13] M. Spira, A. Djouadi, D. Graudenz, and P.M. Zerwas, Nucl. Phys. B453, 17 (1995) and references therein. 